\title{
Multiple UAV-Borne IRS-Aided Millimeter Wave Multicast Communications: A Joint Optimization Framework
}

\author{
Kang Guo, Chao Wang, Member, IEEE, Zan Li, Senior Member, IEEE, Derrick Wing Kwan Ng, Fellow, IEEE, \\ and Kai-Kit Wong, Fellow, IEEE
}

\begin{abstract}
In this letter, we design a resource allocation algorithm for commmunications in millimeter wave (mmWave) multicast systems adopting multiple unmanned aerial vehicle (UAV)borne intelligent reflecting surfaces (IRSs). Considering the effect of blockages of building, we jointly optimize the placement of UAVs and the beamforming at the ground base station (BS) and the passive beamforming at the IRSs for maximizing the minimum rate of multiple user clusters. For handling the nonconvex optimization problem, we first employ the simulated annealing (SA)-based hybrid particle swarm optimization (HPSO) algorithm to design the deployment of UAVs for maximizing the average minimum achievable rate. Then, we propose a penaltybased block coordinated descent (BCD) algorithm to design the active and passive beamforming for maximizing the instantaneous minimum rate. Simulation results validate the efficiency of our proposed joint optimization framework.
\end{abstract}

Index Terms-Intelligent reflecting surface, millimeter wave communication, multicast communications, particle swarm optimization.

\section{INTRODUCTION}

Millimeter wave (mmWave) communication has been regarded as a promising technology for beyond fifth-generation (B5G) communications [1]. However, mmWave communication suffers from severe penetration losses and its performance is susceptible to blockage [1]. Therefore, for improving reliability, one way is to design effective relaying schemes for establishing indirect line-of-sight (LOS) mmWave links to mitigate the effect of blockages. Meanwhile, unmanned aerial vehicle (UAV) communication has also received increasing attention due to its flexible deployment. Compared with traditional terrestrial relay stations, UAVs can operate at high altitudes for avoiding obstacles and establishing a strong LOS link with ground network nodes [2].

Conventionally, relaying techniques operate in a half-duplex (HD) manner that reduces spectral efficiency. Even if fullduplex (FD) relaying techniques are adopted, the performance of active relaying techniques still suffers from severe selfinterference and noise contamination [3]. Recently, intelligent reflecting surface (IRS) has been proposed as a cost effective alternative to existing communication systems. In particular, an IRS is capable of customizing the communication environment

K. Guo, C. Wang, and Z. Li are all with the Integrated Service Networks Lab, Xidian University, Xi'an 710071, China (e-mail: drchaowang@ 126.com).

D. W. K. Ng is with the School of Electrical Engineering and Telecommunications, University of New South Wales, Sydney, 2052, Australia (w.k.ng@unsw.edu.au).

Kai-Kit Wong is with the Department of Electronic and Electrical Engineering, University College London, London WC1E 6BT, U.K. (e-mail: kaikit.wong@ucl.ac.uk). smartly through adjusting the phase shifts of the incident signals via a vast number of passive reflecting elements [4]. To further unlock its potential, the concept of UAV-borne IRS has been proposed which aims to combine the benefits of UAV and IRS communications. Since UAV-borne IRS enjoys flexible deployment and a rich passive beamforming gain, it has generated great interest recently [5]-[7]. However, these works only considered unicast communications and their results are not applicable to the case of multiple UAV-assisted multicast communications. More importantly, the optimization of the UAV's deployment in those works did not take into account the shadowing effect incurred by obstacles. Therefore, existing results, e.g. [6], [7], on optimizing the deployment of multiple UAVs cannot always establish strong LOS links for mmWave communications.

In this letter, we consider UAV-borne IRS-aided mmWave multicasting communications, where multiple UAV-borne IRSs serve multiple user clusters. Considering the effect of blockages of building, we jointly optimize the analog beamforming of the ground base station (BS), the passive beamforming of the IRSs, and the deployment of multiple UAVs for maximizing the minimum achievable rate of multiple users. The design problem is nonconvex due to the coupling among optimization variables and the unit modulus constraints on the beamfomers. To handle the challenges, we firstly derive an upper bound of the average user's rate to obtain a more tractable problem formulation. Then, we propose a simulated annealing (SA)based hybrid particle swarm optimization (HPSO) to optimize multiple UAVs' placement for establishing an indirect LOS link from the BS to multiple users that maximizes the minimum achievable average rate. Once the UAVs' positions are obtained, we propose a penalty-based block coordinate descent (BCD) algorithm to locate an efficient solution of the joint beamforming design. Simulation results validate the effectiveness of our proposed joint optimization framework.

Notations: $(\cdot)^{T},(\cdot)^{*}$, and $(\cdot)^{H}$ denote the transpose, conjugate, and conjugate transpose of a vector, respectively. diag (a) denotes a diagonal matrix whose diagonal elements are from a. $|\cdot|,\|\cdot\|_{2}$, and $\|\cdot\|_{F}$ denote the complex modulus of the element, the Euclidean norm of a vector, and the Frobenius norm of a matrix, respectively. $\arctan (\cdot)$ is the arctangent function. $\mathbb{E}(\cdot)$ is the expectation operator. $\operatorname{Re}(\cdot)$ denotes the real part of a complex-valued variable. $\otimes$ denotes the Kronecker product operation. $\mathbf{x} \sim \mathcal{C N}(\boldsymbol{\Lambda}, \boldsymbol{\Delta})$ denotes the circularly symmetric complex Gaussian vector with mean vector $\boldsymbol{\Lambda}$ and covariance matrix $\boldsymbol{\Delta} . U[0,1]$ and $N[0,1]$ represent a random variable uniformly distributed of $[0,1]$ and a random variable normally 


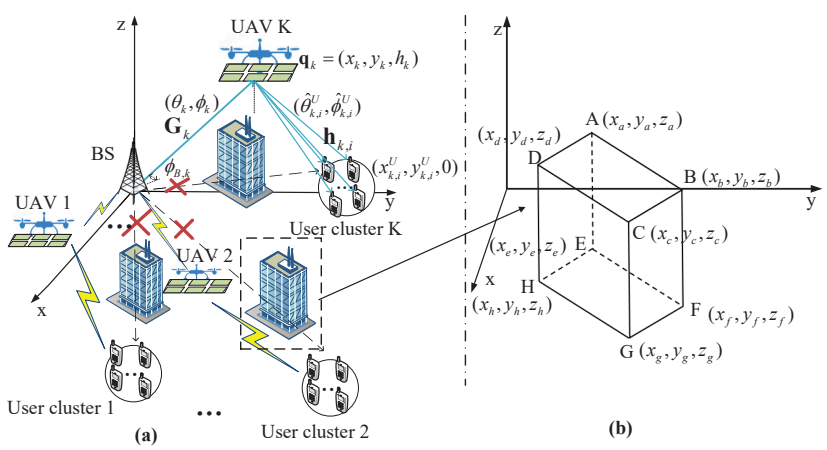

Fig. 1. (a) UAV-borne IRS-aided mmWave multicast communications; (b) illustration of buliding's vertices.

distributed with mean 0 and variance 1 , respectively.

\section{System Model And Problem Formulation}

\section{A. System Model}

Fig. 1(a) shows a typical mmWave multicast communication scenario, where a mmWave BS broadcasts a common signal to $K$ user clusters and the $k$ th user cluster consists of $W_{k}$ users. However, due to the presence of buildings, the direct links between the BS and multiple user clusters are blocked. As a remedy, for establishing effective indirect LOS links from the BS to the users, $K$ UAV-borne IRSs are deployed to assist the communication, where the $k$ th IRS only serves the $k$ th user cluster. For ease of illustration, we denote the $i$ th user of the $k$ th user cluster and the $k$ th IRS as $\mathrm{U}_{k, i}$ and $\mathrm{IRS}_{k}$, respectively, where $k \in \mathcal{K}, i \in \mathcal{W}_{k}, \mathcal{K} \triangleq\{1, \ldots, K\}, \mathcal{W}_{k} \triangleq\left\{1, \ldots, W_{k}\right\}$. In practical scenarios, user clusters are usually far apart from each other. Considering the high path loss and blockage effects, the signals reflected by other $K-1$ UAV-borne IRSs are neglected when considering the signal received at a user cluster. Besides, since we consider multicast communication, the reflected signals from other IRSs can only improve the signal reception. In other words, the adopted assumption establishes a system performance lower bound. On the other hand, the BS is equipped with a single radio frequency (RF) chain and a uniform linear array (ULA) employing $N$ antennas while each UAV-borne IRS is equipped with a uniform planar array (UPA) employing $M=M_{x} \times M_{y}$ elements. The signal received at $\mathrm{U}_{k, i}$ is given by

$$
y_{U_{k, i}}=\sqrt{P} \mathbf{h}_{k, i}^{H} \boldsymbol{\Theta}_{k} \mathbf{G}_{k} \mathbf{f} x+n_{k, i},
$$

where $x \in \mathcal{C N}(0,1)$ denotes the common signal transmitted from the BS, $P$ is the transmitted power, and $n_{k, i} \in \mathcal{C N}\left(0, \sigma^{2}\right)$ is the received noise at $\mathrm{U}_{k, i}$. In addition, $\mathbf{h}_{k, i} \in \mathbb{C}^{M \times 1}$ is the channel vector from UAV-borne $\mathrm{IRS}_{k}$ to its user $\mathrm{U}_{k, i}$, and $\mathbf{G}_{k} \in \mathbb{C}^{M \times N}$ denotes the channel matrix from the BS to UAV-borne $\mathrm{IRS}_{k}$. Furthermore, $\mathbf{f} \in \mathbb{C}^{N \times 1}$ is the analog beamforming vector of the BS and the phase shift matrix of UAV-borne $\mathrm{IRS}_{k}$ is denoted by $\boldsymbol{\Theta}_{k}=$ $\operatorname{diag}\left(\psi_{k, 1} e^{j \theta_{k, 1}}, \psi_{k, 2} e^{j \theta_{k, 2}}, \ldots, \psi_{k, M} e^{j \theta_{k, M}}\right)$, where $\psi_{k, m} \in$ $[0,1]$ and $\theta_{k, m} \in[0,2 \pi)$ denote the reflection amplitude and phase shift of the $m$ th element of UAV-borne $\operatorname{IRS}_{k}$, respectively. Similar to [4], we consider an idealistic reflection model, i.e., $\psi_{k, m}=1$.

\section{B. Channel Model}

In this letter, similar to [5], we adopt the Rician fading channel model, which is given by

$$
\begin{gathered}
\mathbf{G}_{k}=\sqrt{\beta_{\mathrm{B}, k}}\left(\sqrt{\frac{\kappa_{\mathrm{B}, k}}{\kappa_{\mathrm{B}, k}+1}} \mathbf{G}_{k}^{\mathrm{LOS}}+\sqrt{\frac{1}{\kappa_{\mathrm{B}, k}+1}} \mathbf{G}_{k}^{\mathrm{NLOS}}\right), \\
\mathbf{h}_{k, i}=\sqrt{\beta_{k, i}}\left(\sqrt{\frac{\kappa_{k, i}}{\kappa_{k, i}+1}} \mathbf{h}_{k, i}^{\mathrm{LOS}}+\sqrt{\frac{1}{\kappa_{k, i}+1}} \mathbf{h}_{k, i}^{\mathrm{NLOS}}\right),
\end{gathered}
$$

where $\beta_{x, y},\{x, y\} \in\{\{\mathrm{B}, k\},\{k, i\}\}$ denotes the distancedependent path losses. Furthermore, $\mathbf{G}_{k}^{\mathrm{NLOS}}$ and $\mathbf{h}_{k, i}^{\mathrm{NLOS}} \mathrm{de}-$ note the non-LOS (NLOS) components with elements following $\mathcal{C N}(0,1) . \kappa_{x, y}$ denotes the Rican factors. Generally, $\kappa_{x, y} \gg 1$ holds, since the LOS components $\mathbf{G}_{k}^{\mathrm{LOS}}$ and $\mathbf{h}_{k, i}^{\mathrm{LOS}}$ dominate the NLOS channels of the BS-IRS and IRS-User links.

As illustrated in Fig. 1(a), without loss of generality, the BS locates at the origin and the coordinates of UAV-borne IRS $_{k}$ are denoted by $\mathbf{q}_{k}=\left(x_{k}, y_{k}, h_{k}\right)$, with $h_{k, \min } \leq$ $h_{k} \leq h_{k, \max }$ representing the UAV's flight altitude constraint. Besides, $\mathrm{U}_{k, i}$ 's position is denoted as $\left(x_{k, i}^{U}, y_{k, i}^{U}, 0\right)$. The LOS component of the channel matrix $\mathbf{G}_{k}$ can be expressed as

$$
\mathbf{G}_{k}^{\mathrm{LOS}}=e^{-j \frac{2 \pi d_{\mathrm{B}, k}}{\lambda}} \hat{\mathbf{a}}\left(\theta_{k}, \phi_{k}\right) \mathbf{a}^{H}\left(\phi_{\mathrm{B}, k}\right),
$$

where $d_{\mathrm{B}, k}$ denotes the transmission distance between the BS and UAV-borne $\operatorname{IRS}_{k}, \phi_{\mathrm{B}, k}=\arctan \frac{h_{k}}{\sqrt{x_{k}^{2}+y_{k}^{2}}}$ is the angle of departure (AoD) of the signal from the $\mathrm{BS}$ to UAV-borne $\mathrm{IRS}_{k}, \theta_{k}=\arctan \frac{\sqrt{x_{k}^{2}+y_{k}^{2}}}{h_{k}}$ and $\phi_{k}=\arctan \frac{y_{k}}{x_{k}}$ denote the elevation and azimuth angles of arrival (AoA) for the communication from the BS to UAV-borne $\mathrm{IRS}_{k}$, respectively. In addition, $\mathbf{a}\left(\phi_{\mathrm{B}, k}\right)$ and $\hat{\mathbf{a}}\left(\theta_{k}, \phi_{k}\right)$ represent the transmit array response of the $\mathrm{BS}$ and the receive array response of UAVborne $\operatorname{IRS}_{k}$, respectively, which are given by

$$
\begin{aligned}
& \mathbf{a}\left(\phi_{\mathrm{B}, k}\right)=\left[1, e^{-j 2 \pi \frac{d}{\lambda} \sin \left(\phi_{\mathrm{B}, k}\right)}, \cdots, e^{-j 2 \pi(N-1) \frac{d}{\lambda} \sin \left(\phi_{\mathrm{B}, k}\right)}\right]^{T}, \\
& \hat{\mathbf{a}}\left(\theta_{k}, \phi_{k}\right)=\left[1, e^{-j 2 \pi \frac{d}{\lambda} \Phi_{k}\left(\theta_{k}, \phi_{k}\right)}, \cdots, e^{-j 2 \pi \frac{d}{\lambda}\left(M_{x}-1\right) \Phi_{k}\left(\theta_{k}, \phi_{k}\right)}\right]^{T} \\
& \otimes\left[1, e^{-j 2 \pi \frac{d}{\lambda} \Omega_{k}\left(\theta_{k}, \phi_{k}\right)}, \cdots, e^{-j 2 \pi \frac{d}{\lambda}\left(M_{y}-1\right) \Omega_{k}\left(\theta_{k}, \phi_{k}\right)}\right]^{T},
\end{aligned}
$$

where $\Phi_{k}\left(\theta_{k}, \phi_{k}\right) \triangleq \sin \left(\theta_{k}\right) \cos \left(\phi_{k}\right), \quad \Omega_{k}\left(\theta_{k}, \phi_{k}\right) \triangleq$ $\sin \left(\theta_{k}\right) \sin \left(\phi_{k}\right)$, and the antenna spacing $d=\frac{\lambda}{2}, \lambda$ is the wavelength of the carrier. Similarly, the elevation and the azimuth angles of the signal from UAV-borne $\mathrm{IRS}_{k}$ to the $i$ th user are denoted by $\hat{\theta}_{k, i}^{U}=\arctan \frac{\sqrt{\left(x_{k, i}^{U}-x_{k}\right)^{2}+\left(y_{k, i}^{U}-y_{k}\right)^{2}}}{h_{k}}$ and $\hat{\phi}_{k, i}^{U}=\arctan \frac{y_{k, i}^{U}-y_{k}}{x_{k, i}^{U}-x_{k}}$, respectively. Then, the LOS component of the corresponding channel vector can be expressed as

$$
\mathbf{h}_{k, i}^{\operatorname{LOS}}=e^{-j \frac{2 \pi d_{k, i}^{U}}{\lambda}} \hat{\mathbf{a}}\left(\hat{\theta}_{k, i}^{U}, \hat{\phi}_{k, i}^{U}\right),
$$

where $d_{k, i}^{U}$ denotes the transmission distance between UAVborne $\mathrm{IBS}_{k}$ and the $i$ th user, and $\hat{\mathbf{a}}\left(\hat{\theta}_{k, i}^{U}, \hat{\phi}_{k, i}^{U}\right)$ is UAV-borne $\mathrm{IRS}_{k}$ 's array response. 


\section{Problem Formulation}

Since the BS can only acquire the instantaneous CSI corresponding to multiple UAV-borne IRSs through the channel estimation after fixing their positions, following [8], the joint deployment and beamforming optimization is decoupled into the following two problems. The first problem is to optimize the positions of the multiple IRSs based on the statistical CSI, which can be formulated as

$$
\underset{\mathbf{q}_{k} \in \Omega}{\operatorname{maximize}} \mathbb{E}\left(R_{k, i}\right),
$$

where $\Omega$ denotes a feasible deployment area for UAVs. Besides, the achievable rate of the $i$ th user in $k$ th cluster is $R_{k, i} \triangleq \log _{2}\left(1+\frac{P\left|\mathbf{h}_{k, i}^{H} \boldsymbol{\Theta}_{k} \mathbf{G}_{k} \mathbf{f}\right|^{2}}{\sigma^{2}}\right)$. Then, after fixing the positions of UAVs, the BS can adopt existing channel estimation techniques [9] to acquire the instantaneous CSI for facilitating the joint optimization of the active and passive beamforming. Then, the second problem can be formulated as

$$
\begin{array}{ll}
\max _{\mathbf{f}, \boldsymbol{\Theta}_{k}} & \left(\min _{k \in \mathcal{K}, i \in \mathcal{W}_{k}} R_{k, i}\right) \\
\text { s.t. } & \left|\boldsymbol{\Theta}_{k}(m, m)\right|=1, m=1, \cdots, M, \forall k \in \mathcal{K}, \\
& |\mathbf{f}(n)|=1, n=1, \cdots, N .
\end{array}
$$

Solving the above problems is challenging due to the nonconvexity of the deployment optimization and the coupling between the active and passive beamforming in problem (9).

\section{Proposed Optimization Algorithm}

In this section, we propose HPSO-based and BCD-based algorithms to solve problem (8) and problem (9), respectively.

\section{A. Deployment Optimization of Multiple UAV-borne IRSs}

Since the analytical result of $\mathbb{E}\left(R_{k, i}\right)$ is difficult to obtain, as an alternative, similar to [8], we resort to the upper bound of $\mathbb{E}\left(R_{k, i}\right)$ which can be obtained with Jensen's inequality. The corresponding objective function becomes

$$
\mathbb{E}\left(R_{k, i}\right) \leq f\left(\mathbf{q}_{k}\right) \triangleq \log _{2}\left(1+\frac{P N M \mathbb{E}\left(\left\|\operatorname{diag}\left(\mathbf{h}_{k, i}^{H}\right) \mathbf{G}_{k}\right\|_{F}^{2}\right)}{\sigma^{2}}\right) .
$$

Furthermore, given that LOS links are much stronger than NLOS links, we optimize the deployment of multiple IRSs under the constraint that indirect LOS links from the BS to multiple users can be established [6].

As shown in Fig. 1 (b), we approximate the shape of a building by a cuboid with eight vertices, i.e., $A \sim H$. Since mmWave communications rely on synthesizing "pencil-like" beams for compensating high path loss, we assume that there is a direct mmWave link between two nodes when the line connecting them is not blocked [6]. The judging criteria are given as follows. First, the equation of the plane $A B C D$ is given as $M x+N y+O z+P=0$, where $M=\left(y_{b}-y_{a}\right)\left(z_{c}-\right.$ $\left.z_{a}\right)-\left(z_{b}-z_{a}\right)\left(y_{c}-y_{a}\right), N=\left(z_{b}-z_{a}\right)\left(x_{c}-x_{a}\right)-\left(x_{b}-\right.$ $\left.x_{a}\right)\left(z_{c}-z_{a}\right), O=\left(x_{b}-x_{a}\right)\left(y_{c}-y_{a}\right)-\left(y_{b}-y_{a}\right)\left(x_{c}-x_{a}\right), P=$ $-\left(M x_{a}+N y_{a}+O z_{a}\right)$. Then, when $\mathbf{h}_{k, i}$ is blocked by the plane $A B C D$, there exists an intersection point between them. The coordinates of the intersection point can be expressed as $(x, y, z)=\left(k\left(x_{k}-x_{k, i}^{U}\right)+x_{k, i}^{U}, k\left(y_{k}-y_{k, i}^{U}\right)+y_{k, i}^{U}, k h_{k}\right)$, where $k=\frac{-\left(P+M x_{k, i}^{U}+N y_{k, i}^{U}\right)}{M\left(x_{k}-x_{k, i}^{U}\right)+N\left(y_{k}-y_{k, i}^{U}\right)+O h_{k}}$. Furthermore, the intersection point $(x, y, z)$ should satisfy

$$
\left\{\begin{array}{l}
\min \left(x_{a}, x_{b}, x_{c}\right) \leq x \leq \max \left(x_{a}, x_{b}, x_{c}\right) \\
\min \left(y_{a}, y_{b}, y_{c}\right) \leq y \leq \max \left(y_{a}, y_{b}, y_{c}\right) \\
\min \left(z_{a}, z_{b}, z_{c}\right) \leq z \leq \max \left(z_{a}, z_{b}, z_{c}\right)
\end{array}\right.
$$

Since there are five faces formed by a building, when the link has no intersection points with five faces, we can claim that the building does not block the mmWave link.

However, the UAVs' deployment optimization problem is non-convex. Therefore, traditional convex optimization techniques are not applicable. As an alternative, we propose a HPSO-based heuristic algorithm [10] to optimize the deployment of multiple IRSs. In PSO, the solution of optimization problem is called a "particle". Each particle has an initial position and a velocity to determine their next position. The particles follow the current optimal particle and search in the solution space until they converge to an efficient solution. The position and velocity of $t$ th particle at generation $l$ are denoted as $\mathbf{X}_{t}^{l}$ and $\mathbf{V}_{t}^{l}$, respectively. Each particle's best historical position and the best historical position of the entire swarm are denoted as $\mathbf{P}_{t}$ and $\mathbf{P}_{g}$, respectively. The new velocity and position for the next generation can be calculated by

$$
\begin{aligned}
& \mathbf{V}_{t}^{l+1}=w \mathbf{V}_{t}^{l}+c_{1} r_{1}\left(\mathbf{P}_{t}-\mathbf{X}_{t}^{l}\right)+c_{2} r_{2}\left(\mathbf{P}_{g}-\mathbf{X}_{t}^{l}\right), \\
& \mathbf{X}_{t}^{l+1}=\mathbf{X}_{t}^{l}+\mathbf{V}_{t}^{l+1},
\end{aligned}
$$

where $w$ is the inertial factor, $c_{1}$ and $c_{2}$ are accelerated constants, $r_{1}$ and $r_{2}$ are random numbers in the interval [0,1]. For handling a constrained optimization problem, traditional PSO resorts to the penalty-based function method. However, its performance is significantly affected by the penalty factor and the optimal penalty factor is usually challenging to acquire. Hence, we adopt a feasibility-based rule to update the solutions via an iterative process of HPSO, which requires no penalty factor and focuses on guiding the swarm into the feasible set [10]. In particular, the rule can be described as the following two points: (1) Confronting with feasible solutions and infeasible solutions, the former should be chosen; (2) Confronting with two feasible solutions, the one which owns a higher objective function value should be chosen. According to the feasibilitybased rule, $\mathbf{P}_{t}$ will be replaced by $\mathbf{X}_{t}^{l+1}$ under the following two cases: 1) $\mathbf{P}_{t}$ is infeasible, but $\mathbf{X}_{t}^{l+1}$ is feasible; 2) Both $\mathbf{P}_{t}$ and $\mathbf{X}_{t}^{l+1}$ are feasible and $f\left(\mathbf{X}_{t}^{l+1}\right)>f\left(\mathbf{P}_{t}\right)$.

Similarly, $\mathbf{P}_{g}$ is updated based on the rule above at each iteration. Yet, there is a drawback of HPSO that the converged result may be a local optimum. Therefore, the simulated annealing method is utilized to escape from it [10]. In particular, an SA-based search for $\mathbf{P}_{g}$ can be described as follows:

1) Let $j=1, \mathbf{P}_{g}^{\prime}=\mathbf{P}_{g}$; 2) Calculate a new solution by $\mathbf{X}^{\prime}=\mathbf{P}_{g}^{\prime}+\eta \times N(0,1)$, where $\eta$ denotes the step size; 3) Calculate the acceptable probability of a new solution, $p$ : (a) $\mathbf{X}^{\prime}$ is feasible but $\mathbf{P}_{g}^{\prime}$ is infeasible, $p=1$; (b) Both $\mathbf{X}^{\prime}$ and $\mathbf{P}_{g}^{\prime}$ are infeasible, $p=0$; (c) $\mathbf{X}^{\prime}$ is infeasible but $\mathbf{P}_{g}^{\prime}$ is feasible, $p=0$; (d) Both $\mathbf{X}^{\prime}$ and $\mathbf{P}_{g}^{\prime}$ are feasible, 


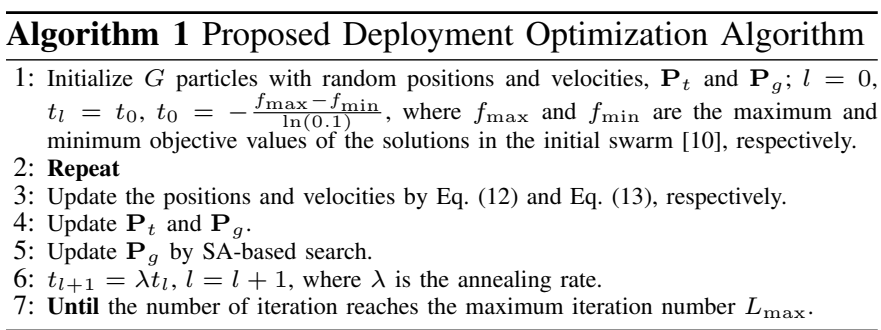

$p=\min \left\{1, \exp \left[\left(f\left(\mathbf{X}^{\prime}\right)-f\left(\mathbf{P}_{g}^{\prime}\right)\right) / t_{l}\right]\right\}$, where $t_{l}$ is the annealing temperature at generation $l$; 4) If $p \geq \alpha$ which is a random variable following $U(0,1), \mathbf{P}_{g}^{\prime}=\mathbf{X}^{\prime}$. 5) $j=j+1$, if $j \leq L$, where $L$ is number of iterations at each generation, go to 2), else stop $\mathbf{P}_{g}=\mathbf{P}_{g}^{\prime}$.

\section{B. Joint Active and Passive Beamforming Optimization}

After obtaining the locations of the UAV-borne IRSs, we jointly optimize the analog beamforming of the BS and the passive beamforming of multiple IRSs to maximize $\min \left\{R_{k, i}\right\}$. By introducing an auxiliary variable $r$, the optimization problem can be reformulated as

$$
\begin{aligned}
\underset{r, \mathbf{f}, \boldsymbol{\Theta}_{k}}{\operatorname{maximize}} & r \\
\text { s.t. } & R_{k, i} \geq r, \forall k \in \mathcal{K}, i \in \mathcal{W}_{k},(9 b),(9 c) .
\end{aligned}
$$

The difficulty for solving the problem above lies in the coupling between $\mathbf{f}$ and $\boldsymbol{\Theta}$ in $R_{k, i}$ and the unit-modulus constraints. By introducing auxiliary optimization variables $X_{k, i}$ and the penalty factor $\rho>0$, we propose a penalty-based algorithm to address problem (14). In particular, problem (14) can be rewritten as

$$
\begin{aligned}
& \underset{r, \mathbf{f}, \boldsymbol{\Theta}_{k}, X_{k, i}}{\operatorname{minimize}_{\rho}}-r+\frac{1}{\rho} \sum_{k \in \mathcal{K}} \sum_{i \in \mathcal{W}_{k}}\left|\mathbf{h}_{k, i}^{H} \boldsymbol{\Theta}_{k} \mathbf{G}_{k} \mathbf{f}-X_{k, i}\right|^{2} \\
& \text { s.t. } \quad \log _{2}\left(1+\frac{P\left|X_{k, i}\right|^{2}}{\sigma^{2}}\right) \geq r, \forall k \in \mathcal{K}, i \in \mathcal{W}_{k},(9 b), \\
&(9 c), \mathbf{h}_{k, i}^{H} \boldsymbol{\Theta}_{k} \mathbf{G}_{k} \mathbf{f}=X_{k, i}, \forall k \in \mathcal{K}, i \in \mathcal{W}_{k} .
\end{aligned}
$$

We apply the BCD algorithm to obtain a stationary solution of problem (15) by updating the optimization variables iteratively. In particular, the optimization variables of (15) can be divided into three blocks: 1) passive beamformers of multiple IRSs, $\left.\boldsymbol{\Theta}_{k} ; 2\right)$ analog beamforming vector of the BS, f; 3) auxiliary variables, $r$ and $X_{k, i}$. Then, we optimize each block of variables by fixing the other ones iteratively. In this regard, the non-convex joint design problem can be decoupled into the following three problems:

1) Fixing $\mathbf{f}, r, X_{k, i}$, we first consider the optimization of the passive beamformer of each UAV-borne IRS. By denoting $\mathbf{v}_{k}$ as a vector composed of the diagonal elements of $\boldsymbol{\Theta}_{k}$, we have $\mathbf{h}_{k, i}^{H} \boldsymbol{\Theta}_{k} \mathbf{G}_{k} \mathbf{f}=\mathbf{v}_{k}^{H} \mathbf{d}_{k, i}$ where $\mathbf{d}_{k, i}=\operatorname{diag}\left(\mathbf{h}_{k, i}^{H}\right) \mathbf{G}_{k} \mathbf{f}$. Then, the passive beamforming optimization can be formulated as

$$
\begin{aligned}
\underset{\mathbf{v}_{k}}{\operatorname{minimize}} & \sum_{k \in \mathcal{K}} \sum_{i \in \mathcal{W}_{k}}\left|\mathbf{v}_{k}^{H} \mathbf{d}_{k, i}-X_{k, i}\right|^{2} \\
\text { s.t. } & \left|\mathbf{v}_{k}(m)\right|=1, \forall m=1, \cdots, M, \forall k \in \mathcal{K} .
\end{aligned}
$$

Since the objective function is decomposable, multiple passive beamformers can be optimized in parallel. In the following, we

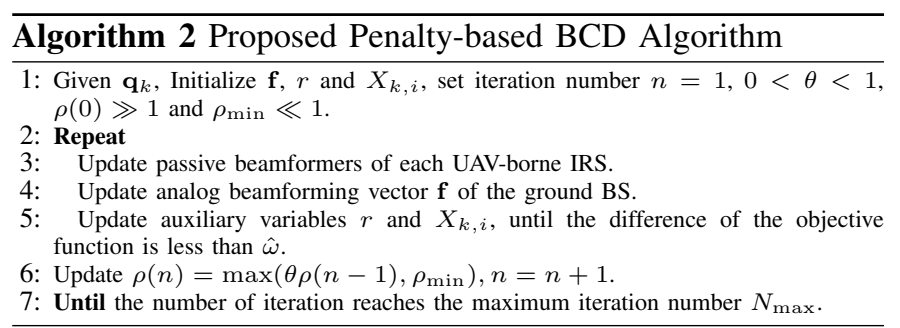

propose an iterative method to optimize $\mathbf{v}_{k}$. Specifically, by fixing $\mathbf{v}_{k}\left(m^{\prime}\right), \forall m^{\prime} \neq m, m^{\prime} \in \mathcal{M}$, where $\mathcal{M} \triangleq\{1, \ldots, M\}$, we notice that the objective function of (16) with respect to $\mathbf{v}_{k}(m)$ can be written as

$$
2 \operatorname{Re}\left\{\mathbf{v}_{k}(m) u_{m}\right\}+\sum_{m^{\prime} \neq m n \neq m}^{M} \sum_{n \neq m}^{M} \mathbf{R}\left(m^{\prime}, n\right) \mathbf{v}_{k}\left(m^{\prime}\right) \mathbf{v}_{k}^{H}(n)+C,
$$

where

$$
\begin{aligned}
& u_{m}=\sum_{m^{\prime} \neq m}^{M} \mathbf{R}\left(m, m^{\prime}\right) \mathbf{v}_{k}^{H}\left(m^{\prime}\right)-\mathbf{b}(m), \\
& \mathbf{R}=\sum_{i \in \mathcal{W}_{k}} \mathbf{d}_{k, i} \mathbf{d}_{k, i}^{H}, \mathbf{b}=\sum_{i \in \mathcal{W}_{k}} \mathbf{d}_{k, i} X_{k, i}^{H}, \\
& C=\mathbf{R}(m, m)-2 \operatorname{Re}\left\{\sum_{m^{\prime} \neq m}^{M} \mathbf{v}_{k}\left(m^{\prime}\right) \mathbf{b}\left(m^{\prime}\right)\right\}+\sum_{k \in \mathcal{K}}\left|X_{k, i}\right|^{2} .
\end{aligned}
$$

Fixing other phase-shifters, the value of (17) is minimized with $\mathbf{v}_{k}(m)=-\frac{u_{m}^{*}}{\left|u_{m}\right|}, \forall m$. Employing this strategy, we can optimize $\mathbf{v}_{k}(m)$ sequentially, until the convergence is achieved.

2) Fixing $\boldsymbol{\Theta}_{k}, r, X_{k, i}$, we then consider the optimization of analog beamforming vector $\mathbf{f}$, which is formulated as

$$
\underset{\mathbf{f}}{\operatorname{minimize}} \sum_{k \in \mathcal{K}} \sum_{i \in \mathcal{W}_{k}}\left|\mathbf{h}_{k, i}^{H} \boldsymbol{\Theta}_{k} \mathbf{G}_{k} \mathbf{f}-X_{k, i}\right|^{2} \text {, s.t.(9c). }
$$

Following the procedures given in [11], the problem above can be handled by the manifold optimization algorithm.

3) Fixing $\boldsymbol{\Theta}_{k}$, f, the joint optimization of $r$ and $X_{k, i}$ can be formulated as

$$
\begin{aligned}
\underset{r, X_{k, i}}{\operatorname{minimize}} & -r+\frac{1}{\rho} \sum_{k \in \mathcal{K}} \sum_{i \in \mathcal{W}_{k}}\left|\mathbf{h}_{k, i}^{H} \boldsymbol{\Theta}_{k} \mathbf{G}_{k} \mathbf{f}-X_{k, i}\right|^{2} \\
\text { s.t. } & \log _{2}\left(1+\frac{P\left|X_{k, i}\right|^{2}}{\sigma^{2}}\right) \geq r, \forall k \in \mathcal{K}, i \in \mathcal{W}_{k} .
\end{aligned}
$$

However, problem (18) is nonconvex due to the non-convex constraint (18b). To tackle this, we use the widely adopted concave-convex procedure to approximate it by a sequence of convex problems. In particular, a subset of constraint (18b) is given by its first-order Taylor expansion as

$$
-\left|X_{k, i}(l-1)\right|^{2}+2 \operatorname{Re}\left(X_{k, i}^{H}(l-1) X_{k, i}\right) \geq \frac{\left(2^{r}-1\right) \sigma^{2}}{P},
$$

where $X_{k, i}(l-1)$ is the optimal solution of the convex approximation problem at the $(l-1)$ th iteration of this subproblem.

\section{Simulation Results}

In this section, we evaluate the performance of our proposed joint optimization algorithm. The network parameters are given as follows. The carrier frequency is set as $28 \mathrm{GHz}$, path loss $\beta(D)$ is $61.4+20 \log _{10}(D) \mathrm{dB}$ [2], and Rician fading 
TABLE I

VERTICES OF MUlTiPLE BUILDings.

\begin{tabular}{|c|c|}
\hline \multirow{2}{*}{ Building I } & $\begin{array}{c}(5,5,0),(5,15,0),(-5,15,0),(-5,5,0) \\
\end{array}$ \\
\hline \multirow{2}{*}{ Building II } & $(15,15),(5,15,15),(-5,15,15),(-5,5,15)$ \\
& $(15,15,18),(15,25,0),(10,25,0),(10,15,0)$ \\
\hline \multirow{2}{*}{ Building III } & $(30,10,0),(30,20,0),(20,25,18),(10,15,18)$ \\
& $(30,10,18),(30,20,18),(20,20,18),(20,10,18)$ \\
\hline
\end{tabular}

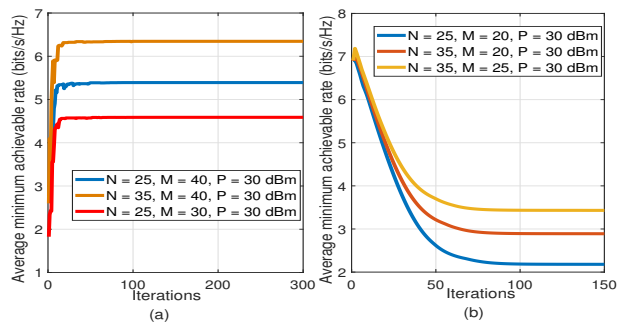

Fig. 2. Convergence of proposed algorithms, (a) Alogrithm 1; (b) Alogrithm

factors $\kappa_{\mathrm{B}, k}=\kappa_{k, i}=30 \mathrm{~dB}$. The noise power is set as $\sigma^{2}=-110 \mathrm{dBm}$. As shown in Fig. $1, K=3, W_{k}=4$, $h_{k, \min }=20 \mathrm{~m}, h_{k, \max }=50 \mathrm{~m}$, the centers of three user clusters are $(0,50),(30,40)$, and $(40,30)$ respectively, and the coordinates of vertices for each building are given in Table I. For Algorithm 1, we set $c_{1}=c_{2}=2, G=300, L_{\max }=300$, $L=20, \lambda=0.94, \eta=0.001$, and $\omega$ linearly decreases from 0.9 to 0.4 [10]. For Algorithm 2, we set $\theta=0.9, \rho_{\min }=10^{-5}$, $\hat{\omega}=10^{-3}$ and $N_{\max }=150$. For showing the superiority of Algorithm 2, we consider a baseline scheme to design the beamformers, where the phase shifts of IRSs are aligned with the channel vector of the farthest user in each user cluster, for guaranteeing users' fairness. Then, the baseline scheme firstly optimizes the BS's virtual full-digital beamforming vector $\hat{\mathbf{f}}$ to maximize the achievable minimum rate and recovers BS's analog beamforming $\mathbf{f}$ by minimizing $\|\mathbf{f}-\hat{\mathbf{f}}\|_{F}^{2}$.

Fig. 2 shows the convergence rate of our proposed algorithms. We can find that the convergence rate of the proposed Algorithms 1 and 2 remains almost unchanged for different $M$ and $N$ showing our proposed algorithms enjoy an excellent scalability. Fig. 3 shows the average minimum achievable rate versus the number of IRS reflecting elements, $M$. From Fig. 3, we observe that our proposed algorithm can achieve significant performance gains compared with the baseline scheme due to the proposed optimization framework. Besides, the minimum achievable rate of $K$ user clusters increases with the increasing $M$, which is due to the increasing design degrees-of-freedom (DoF) brought by increasing $M$. However, the increasing rate decreases with the increasing $M$, which is due to the channel hardening effects in the link from the BS to the IRS. In contrast, the minimum achievable rate of the baseline scheme keeps almost the same with increasing $M$, as its information beam does not always align with the best direction for improving the system performance.

\section{Conclusions}

This letter established a joint optimization framework to design the deployment of multiple UAV-borne IRSs, the passive

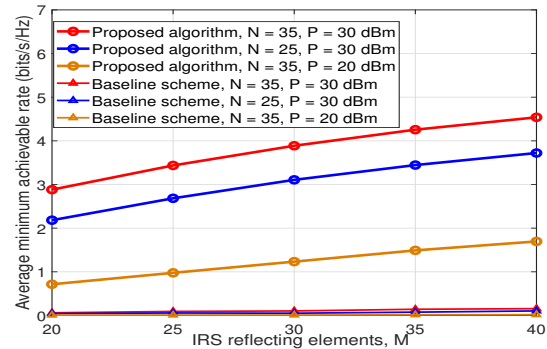

Fig. 3. Average minimum achievable rate versus IRS reflecting elements, $M$.

beamforming, and the analog beamforming of the mmWave $\mathrm{BS}$ for maximizing the minimum achievable rate of the UAVborne IRS-aided mmWave multicast communications. We first decomposed the considered joint optimization problem into the deployment optimization and the joint beamforming optimization problems. Then, we proposed a HPSO-based heuristic algorithm to optimize the deployment of multiple UAVborne IRSs for maximizing the achievable rate averaging over the statistical CSI. After obtaining the positions of multiple IRSs, we proposed a penalty-based BCD algorithm to jointly optimize the beamformers of multiple UAV-borne IRSs and the mmWave BS for maximizing the instantaneous minimum achievable rate. Simulation results verify the efficiency of our proposed joint optimization framework compared with a baseline scheme.

\section{REFERENCES}

[1] S. Rangan, T. S. Rappaport, and E. Erkip, "Millimeter-wave cellular wireless networks: Potentials and challenges," Proc. IEEE, vol. 102, no. 3, pp. 366-385, Mar. 2014

[2] L. Zhang, H. Zhao, S. Hou, Z. Zhao, H. Xu, X. Wu, Q. Wu, and R. Zhang, "A survey on 5G millimeter wave communications for UAVassisted wireless networks," IEEE Access, vol. 7, pp. 117460-117 504, Jul. 2019.

[3] M. D. Renzo, K. Ntontin, J. Song, F. H. Danufane, X. Qian, J. De Rosny, D. T. Phan-Huy, O. Simeone, R. Zhang, M. Debbah, G. Lerosey, M. Fink, S. Tretyakov, and S. Shamai, "Reconfigurable intelligent surfaces vs. relaying: Differences, similarities, and performance comparison," IEEE Open J. Commun. Soc., vol. 1, pp. 798-807, Jun. 2020.

[4] Q. Wu and R. Zhang, "Towards smart and reconfigurable environment: Intelligent reflecting surface aided wireless network," IEEE Commun. Mag., vol. 58, no. 1, pp. 106-112, Jan. 2020.

[5] S. Li, B. Duo, X. Yuan, Y. Liang, and M. Di Renzo, "Reconfigurable intelligent surface assisted UAV communication: Joint trajectory design and passive beamforming," IEEE Wireless Commun. Lett., vol. 9, no. 5, pp. 716-720, May 2020.

[6] Q. Zhang, W. Saad, and M. Bennis, "Reflections in the sky: Millimeter wave communication with UAV-carried intelligent reflectors," in Proc. IEEE Global Commun. Conf. (GLOBECOM), 2019, pp. 1-6.

[7] H. Lu, Y. Zeng, S. Jin, and R. Zhang, "Aerial intelligent reflecting surface: Joint placement and passive beamforming design with 3D beam flattening," arXiv preprint arXiv:2007.13295, 2020.

[8] L. Zhu, J. Zhang, Z. Xiao, X. Cao, X. G. Xia, and R. Schober, "Millimeter-wave full-duplex UAV relay: Joint positioning, beamforming, and power control," IEEE J. Sel. Areas Commun., vol. 38, no. 9, pp. 2057-2073, Sept. 2020.

[9] E. Basar, M. D.Renzo, J. De Rosny, M. Debbah, M. Alouini, and R. Zhang, "Wireless communications through reconfigurable intelligent surfaces," IEEE Access, vol. 7, pp. 116753-116773, Aug. 2019.

[10] Q. He and L. Wang, "A hybrid particle swarm optimization with a feasibility-based rule for constrained optimization," Applied Mathematics and Computation, vol. 186, no. 2, pp. 1407-1422, Mar. 2007.

[11] X. Yu, D. Xu, and R. Schober, "MISO wireless communication systems via intelligent reflecting surfaces," in Proc. IEEE/CIC Int. Conf. Commun. China (ICCC), Aug. 2019, pp. 735-740. 